\title{
Screening of differentially expressed genes associated with human glioblastoma and functional analysis using a DNA microarray
}

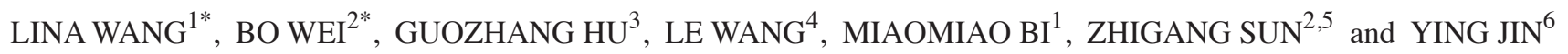 \\ Departments of ${ }^{1}$ Ophthalmology, ${ }^{2}$ Neurosurgery and ${ }^{3}$ Emergency Medicine, China-Japan Union Hospital of Jilin University, \\ Changchun, Jilin 130033; ${ }^{4}$ Department of Ophthalmology, The First Hospital of Jilin University, Changchun, Jilin 130021; \\ ${ }^{5}$ Department of Neurosurgery, Affiliated Hospital of Inner Mongolia University for the Nationalities, Tongliao 028007; \\ ${ }^{6}$ Department of Neurology, Institute of Jilin Oilfield General Hospital, Changchun, Jilin 131200, P.R. China
}

Received June 26, 2014; Accepted March 10, 2015

DOI: $10.3892 / \mathrm{mmr} .2015 .3659$

\begin{abstract}
Glioblastoma multiforme (GBM) is the most malignant type of human glioma, and has a poor prognosis. Screening differentially expressed genes (DEGs) in brain tumor samples and normal brain samples is of importance for identifying GBM and to design specific-targeting drugs. The transcriptional profile of GSE30563, containing three genechips of brain tumor samples and three genechips of normal brain samples, was downloaded from Gene Expression Omnibus to identify the DEGs. The differences in the expression of the DEGs in the two different samples were compared through hierarchical biclustering. The co-expression coefficient of the DEGs was calculated using the information from COXPRESdb, the network of the DEGs was constructed and functional enrichment and pathway analysis were performed. Finally, the transcription factors of important DEGs were predicted. A total of 1,006 DEGs, including 368 upregulated and 638 downregulated DEGs, were identified. A close correlation was demonstrated between six important genes, associated with immune response, HLA-DQB1, HLA-DRB1, HLA-DPA1, HLA-B, HLA-DMA and HLA-DRA, and the immune response. Allograft rejection was selected as the most significant pathway. A total of 17 transcription factors, including nuclear factor (NF)- $\kappa \mathrm{B}$ and NF- $\kappa \mathrm{B} 1$, and their binding sites containing these six DEGs, were also identified. The DEGs, including major histocompatibility complex (MHC) class II, DQ $\beta 1$, MHC class II, DR $\beta 1$, MHC class IB, MHC class II, DM $\alpha$, MHC class II, DP $\alpha 1$, MHC class II, DR $\alpha$, may provide novel targets for the diagnosis and treatment of
\end{abstract}

Correspondence to: Dr Zhigang Sun, Department of Neurosurgery, China-Japan Union Hospital of Jilin University, 126 Xiantai Avenue, Changchun, Jilin 130033, P.R. China

E-mail: sunzhigang0001@hotmail.com

*Contributed equally

Key words: glioblastoma multiforme, co-expression networks, transcription factors
GBM. The transcription factors of these six genes and their binding sites may also provide evidence and direction for identifying target-specific drugs.

\section{Introduction}

Glioblastoma multiforme (GBM) is the most malignant type of human glioma and has a poor prognosis. Despite advances in diagnosis and treatment, the median survival rate of patients with GBM remains $\sim 15$ months (1-3). GBM is one of the most intractable types of refractory tumor. Surgery and radiotherapy have been the predominant forms of therapy for GBM, however, the curative effect is poor (4). Gene treatment offers possible approaches in the treatment of GBM, however, it retains shortcomings, including the lack of special target genes and high-efficiency carriers (5). For decades, how to treat GBM has remained a focus and difficulty in investigations and clinical treatment.

There has been substantial progress in the use of microarrays for investigating the molecular mechanisms of brain gliomas. Microarrays are valuable for identifying the important genes involved in the occurrence, development and targeted therapy of gliomas (6). Microarrays have been used to screen genes associated with $\operatorname{GBM}(3,7,8)$, and bioinformatics analysis has revealed that these genes, screened using a microarray, are closely associated with cell signal transduction, cell metabolization, cytoskeleton and motility, immunity, the cell cycle and apoptosis (7). However, the specific molecular mechanisms underlying human GBM are not yet fully understood.

In the present study, the transcriptional profile of GSE30563 was downloaded and the differentially expressed genes (DEGs) between GBM and healthy brain tissues were identified. In addition, a co-expression network of DEGs was constructed, and Gene Ontology (GO) functional and Kyoto Encyclopedia of Genes and Genomes (KEGG) pathway analyses were performed to identify the target genes for the diagnosis and treatment of GBM. Finally, the interactions between DEGs and transcription factors were assessed for further evaluation at the molecular level. The findings of these investigations may contribute to improvements in the understanding and diagnosis of GBM, and the design of target-specific drugs. 


\section{Materials and methods}

Microarray data. The transcriptional profile of GSE30563 was downloaded from Gene Expression Omnibus (GEO) (http://www.ncbi.nlm.nih.gov/geo/), which was based on the platform of the Affymetrix Human Genome U133 Plus 2.0 array. This dataset (http://www.ncbi.nlm.nih. gov/geo/query/acc.cgi?acc=GSE30563) was deposited by Lee et al (Ajou University School of Medicine, Suwon, South Korea). To identify DEGs, human brain tumor samples and normal brain samples were collected from patients with a brain tumor, for subsequent RNA extraction and hybridization on Affymetrix microarrays. A total of six genechips (GSM758396, GSM758397, GSM758398, GSM758399, GSM758400 and GSM758401) were available for further analysis, including three genechips of brain tumor samples and three genechips of normal brain samples.

Data pre-processing and analysis of differential expression. The probe-level data in the raw data files were converted into expression measures, according to the function of $\log 2(9)$. The expression values of all the probes were matched to the genes and the empty probes were reduced. The LIMMA package in R (http://www.bioconductor.org/packages/release/bioc/html/limma.html) (10) was used to identify the DEGs in the brain tumor samples compared with the healthy controls. The present study selected a cut-off criteria of $\mathrm{P} \leq 0.01$ and $\log 2$ fold changel $>1$ to identify the DEGs.

Comparison of differential expression in different samples. The expression values of the DEGs in each sample were extracted from the expression value files, according to the information of the probes corresponding to the DEGs. Based on these expression values, the pheatmap package in $\mathrm{R}$ was used for hierarchical clustering (11), through Euclidean distance (12), and a heat map was constructed.

Searching for the co-expression network of DEGs. COXPRESdb (http://coxpresdb.hgc.jp) is a database of co-expressed gene networks and can assist in elucidating the function and regulation network of genes in a wide range of mammals (13). Based on the hypothesis that genes, which are regulated by the same transcription factor, are co-expressed, and that co-expressed genes may be associated in function (14), the DEGs were divided into either upregulated or downregulated genes, and co-expression networks of the DEGs were constructed, using the information obtained from COXPRESdb. The gene pairs with a co-expression coefficient $>0.6$ were selected, and the network was visualized using Cytoscape software (http://www.cytoscape.org/) (15).

GO and pathway analyses of the DEGs. The Database for Annotation, Visualization and Integrated Discovery (DAVID) is a web-accessible program, which clusters distinct genes by the pathways in which they are involved, producing intuitive graphical summaries (16). The DEGs in the co-expression networks in the present study were analyzed using DAVID to identify which biological process the genes in the networks were involved in. To circumvent the problem of multi-testing, which may induce too many false positive results, the
Benjamini and Hochberg method (17) was used to adjust the raw P-values into false discovery rate (FDR). FDR $<0.05$ was used as the cut-off criterion. The DEGs were analyzed using KEGG, and the biological pathways, which were mapped significantly by the DEGs were identified $(18,19)$.

Interactions between DEGs and transcription factors. The gene sequence can inhibit or enhance the expression of a gene by covalently binding to the transcription factor DNA binding domain (20). In the present study, based on text mining using PubChem Bioassay neighboring analysis (http://pubchem. ncbi.nlm.nih.gov/) (21), the interactions between the genes and transcription factors were extracted using the online tool, EpiTect ChIP qPCR Primers (http://www.sabiosciences. com/chipqpcrsearch.php?app=TFBS).

\section{Results}

Screening for DEGs. Following data preprocessing, the expression values with high standardization were analyzed through comparison of the differences (Fig. 1). A total of 1,006 DEGs, exhibiting a cut-off criteria $\mathrm{P}<0.01$ and $|\log \mathrm{FCl}|>1$, were selected, including 638 downregulated and 368 upregulated DEGs.

Hierarchical clustering analysis of DEGs. Hierarchical clustering revealed systematic variations in the expression levels of genes between the brain tumor samples and the healthy control samples (Fig. 2). The results revealed that these differential probes were able to distinguish these two groups from the whole samples.

Searching for the co-expression networks of DEGs. COXPRESdb was used to construct a co-expression network of the resulting DEGs, following which a total of 113 gene pairs with a co-expressed coefficient $>0.6$ were identified. These were subsequently visualized using Cytoscape (Fig. 3) and, among them, the ANXA2 and ANXA2P2 gene pairs were selected as exhibiting the highest co-expression coefficient of 0.94 .

GO functional and pathway analyses of the DEGs. Based on the co-expression network, GO functional enrichment analysis of the DEGs was performed using DAVID software, with FDR $<0.05$. The results indicated that 59 DEGs were significantly increased in seven GO terms (Table I). The most significant functional term was associated with the immune response, and the genes enriched in this term included major histocompatibility complex (MHC) class II, DQ $\beta 1$ (HLA-DQB1), MHC class II, DR $\beta 1$ (HLA-DRB1), MHC class IB (HLA-B), MHC class II, DM $\alpha$ (HLA-DMA), MHC class II, DP $\alpha 1$ (HLA-DPA1) and MHC class II, DR $\alpha$ (HLA-DRA). The KEGG pathway analysis identified six pathways (Table II), which had an FDR $<0.05$. The most significant pathway was associated with allograft rejection, and the genes enriched in this pathway were HLA-DQB1, HLA-DRB1, HLA-DPA1, HLA-B, HLA-DMA and HLA-DRA.

Transcription factors of important DEGs. Based on the comparison of the most significant biological process 
Table I. Results of GO functional enrichment analysis for differentially expressed genes.

\begin{tabular}{llrr}
\hline Term & \multicolumn{1}{c}{ Name } & Count & FDR \\
\hline GO:0006955 & Immune response & 18 & $9.03 \mathrm{E}^{-06}$ \\
GO:0019882 & Antigen processing and presentation & 8 & $1.33 \mathrm{E}^{-04}$ \\
GO:0002504 & Polysaccharide antigen via MHC class II & 6 & $5.35 \mathrm{E}^{-04}$ \\
GO:0030198 & Extracellular matrix organization & 7 & 0.011087 \\
GO:0030199 & Collagen fibril organization & 5 & 0.012866 \\
GO:0043062 & Extracellular structure organization & 8 & 0.012897 \\
GO:0002252 & Immune effector process & 7 & 0.04723
\end{tabular}

GO, Gene Ontology; FDR, false discovery rate.

Table II. Results of differentially expressed genes in KEGG pathway enrichment analysis.

\begin{tabular}{lll}
\hline Term & \multicolumn{1}{c}{ KEGG } & FDR \\
\hline hsa05330 & Allograft rejection & 0.008554 \\
hsa05322 & Systemic lupus erythematosus & 0.011522 \\
hsa05332 & Graft-versus-host disease & 0.012834 \\
hsa04940 & Type I diabetes mellitus & 0.018628 \\
hsa04612 & Antigen processing and presentation & 0.046172 \\
hsa05320 & Autoimmune thyroid disease & 0.048764 \\
\hline
\end{tabular}

FDR, false discovery rate; KEGG, Kyoto Encyclopedia of Genes and Genomes.

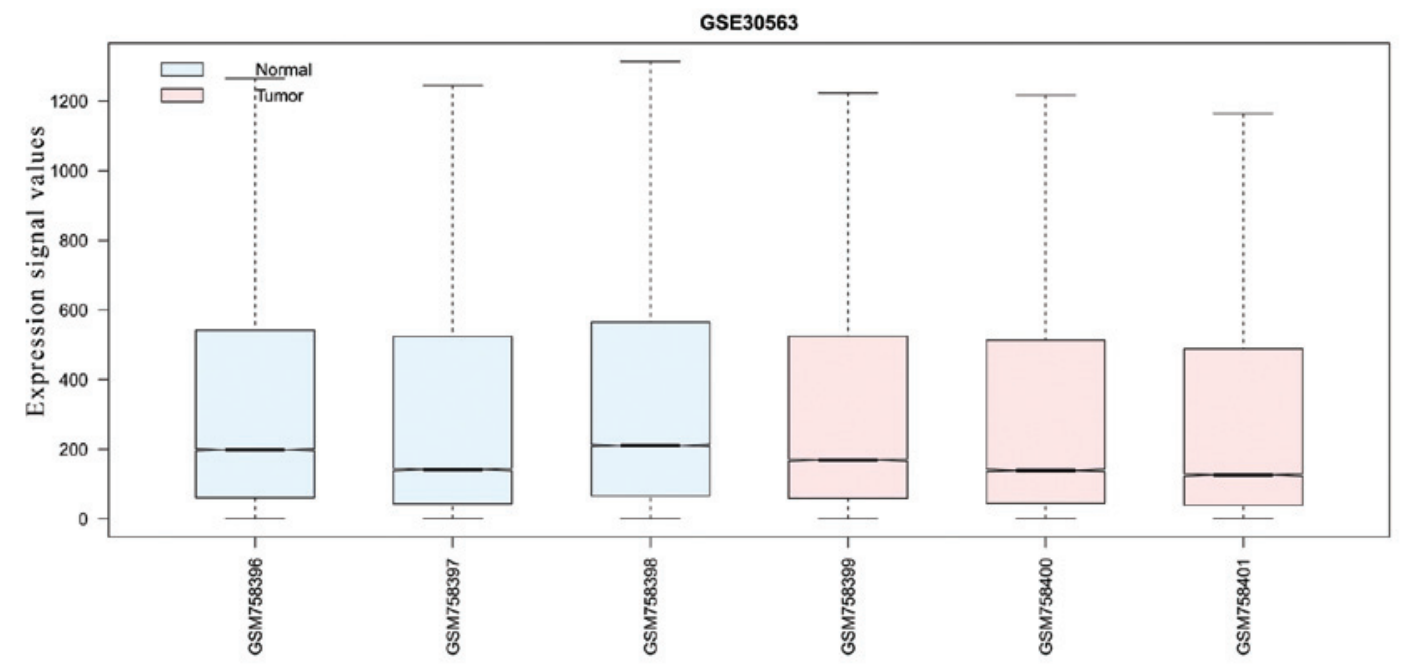

Figure 1. Box-plot of the gene expression values following standardization, detected using the LIMMA package in R. The pink boxes indicate the samples from patients with glioblastoma multiforme and the blue indicates the normal samples. The black line represents the median value in each set of data. The whiskers represent the upper edge and lower edge of the box plot, which are the maximum and minimum expression values, respectively.

and KEGG pathway, six genes, including HLA-DQB1, HLA-DRB1, HLA-DPA1, HLA-B, HLA-DMA and HLA-DRA, were differentially expressed in two lists at the same time, located on the short arm of chromosome 6 (Fig. 4). A total of 17 transcription factors, including nuclear factor $(N F)-\kappa B, N F-\kappa B 1$ and their binding sites, were identified with these six important DEGs.

\section{Discussion}

GBM is one of the most frequent types of human brain cancer and it develops from either a lower grade astrocytic tumor or primary GBM (22). However, neither chemotherapy nor radiotherapy have been effective in treating this type of cancer (23). Biochip technology has emerged as an efficient, rapid and 


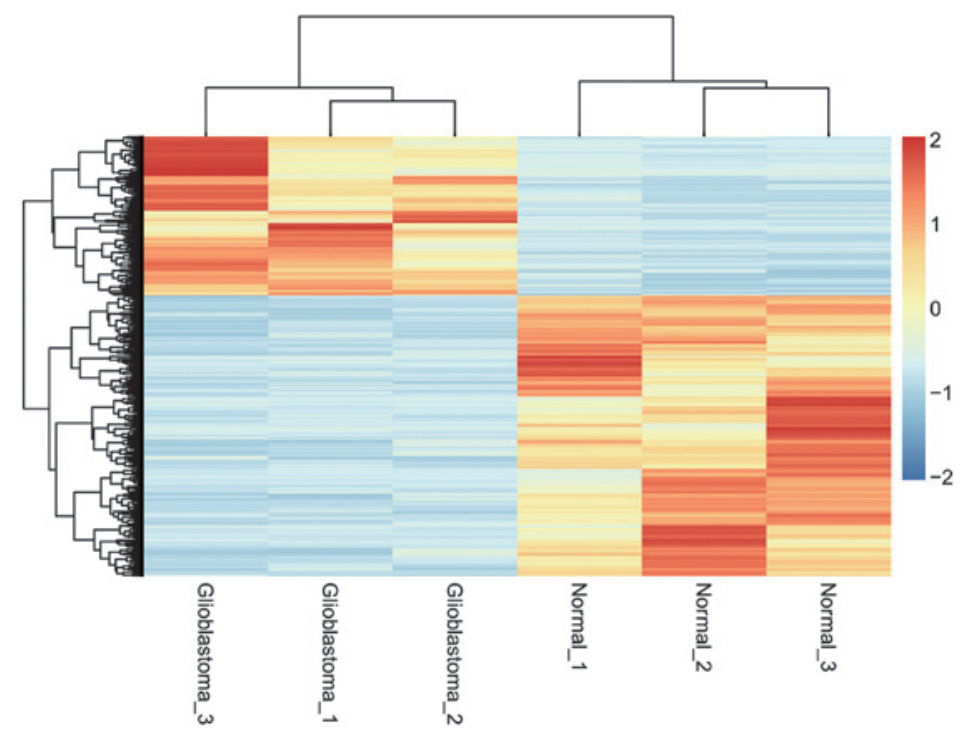

Figure 2. Hierarchical clustering of human brain tumor samples and normal brain tissue samples based on the differentially expressed genes. The Database for Annotation, Visualization and Integrated Discovery programme was used for clustering. Blue indicates the expression value of downregulated genes, orange indicates the expression value of upregulated genes and red boxes represent clustering of the normal brain samples with the brain tumor samples.

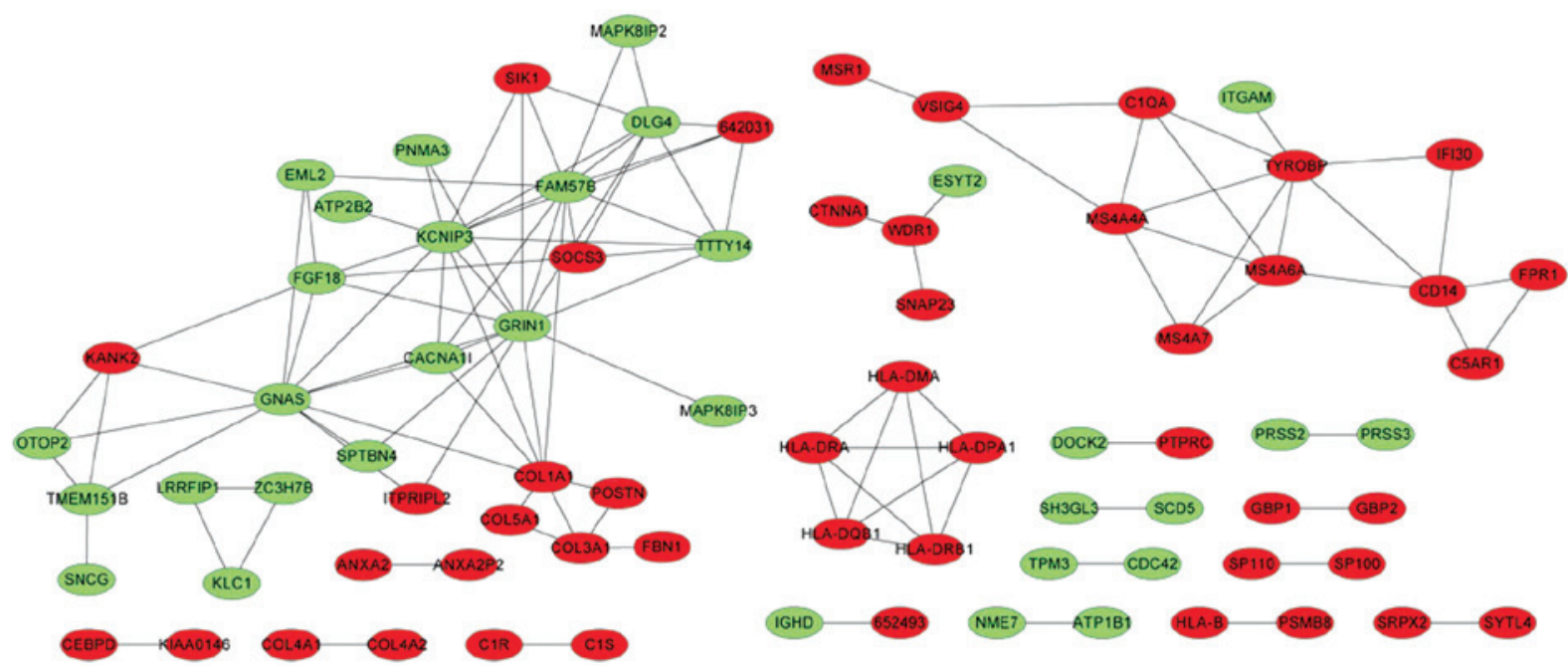

Figure 3. Interaction networks of the DEGs. The red nodes represent the upregulated DEGs and the green nodes represent the downregulated DEGs. DEG, differentially expressed genes.

HLA-DQB1
chr6:32,642,466
NF-kappaB
NF-kappaB1
RelA
HLA-DRB1
chr6:32,547,562
NF-kappaB
NF-kappaB1
CULT1

Figure 4. Diagrammatic representation of the transcription factors of differentially expressed genes and their binding sites with the genes. Red arrows indicate transcription start sites, and green lines represent transcription binding sites. NF, nuclear factor. 
multi-parameter technology, which provides a useful strategy for diagnosis, classification and therapy against the development of human GBM.

The present study identified 1,006 DEGs, including 638 upregulated and 368 downregulated DEGs, from the gene expression profile of GSE30563. Following this, a co-expression network of DEGs was constructed and GO functional and KEGG pathway analyses were performed. Functional analysis of the DEGs demonstrated a close correlation with the immune response. Additionally, allograft rejection was the most significantly enriched pathway, and the genes involved in these processes were HLA-DQB1, HLA-DRB1, HLA-DPA1, HLA-B, HLA-DMA and HLA-DRA.

These genes belong to the human leukocyte antigen (HLA) gene family. The HLA genes encode numerous molecules, including HLA class I and II, and are known to be associated with the majority of autoimmune diseases (24). Human HLA molecules are important in eliminating tumor cells with cellular and humoral immunity $(25,26)$. The HLA-DRA, HLA-DRB1, HLA-DQB1, HLA-DPA1 genes belong to the HLA class II $\beta$ chain paralogs. They bind peptides derived from antigens, which access the endocytic route of antigen presenting cells (APCs) and present them on the cell surface for recognition by CD4 T-cells (27). The HLA-DMA gene belongs to the HLA class II $\alpha$ chain paralogues. It is involved in the peptide loading of MHC class II molecules, by assisting in the release of the class II-associated invariant chain peptide molecule from the peptide binding site (28). The HLA-B gene belongs to the HLA class I heavy chain paralogues. Class I molecules are important in the immune system by presenting peptides derived from the endoplasmic reticulum lumen (29). GBM function profoundly impairs the immune response by inhibiting the proliferation and activation of T-cells, inducing regulator T-cells and triggering apoptosis (30). In addition, it has been reported that the expression of HLA is positively associated with patients with GBM. For example, compared with the control population, HLA-B*27 exhibits a 2.7-fold increase and HLA-DRB1*15 exhibits a 2.2-fold increase in the risk of glioma occurrence (31), suggesting that the HLA family may be used as a specific therapeutic molecular target in the treatment of GBM.

Allograft rejection includes a coordinated response of the innate and adaptive immune systems of the host (32). The mechanism for allograft rejection in the immune response may be similar to this (33), in that the innate immune system is involved in the early phase of the allograft response by chemokines and cell adhesion, which are essential for leukocyte migration into the graft and T-cell trafficking between lymph nodes and the transplant. The T cells and other cells from the innate immune system act synergistically to reject the allograft through nonexclusive pathways, including the cytotoxicity of contact-dependent $T$ cells, the activation of granulocyte and natural killer cells and the production of alloantibody. GBM is closely associated with the immune response, suggesting that allograft rejection is possibly involved in the processes of GBM. However, further investigations are required to confirm this hypothesis.

In conclusion, the present study demonstrated that HLA-DQB1, HLA-DRB1, HLA-DPA1, HLA-B, HLA-DMA, HLA-DRA are associated with GBM. In addition, the patho- genesis of GBM was closely associated with the immune response and pathways, including allograft rejection. These findings may offer novel targets for the diagnosis and treatment of GBM.

\section{Acknowledgements}

The authors would like to thank to all the authors who contributed to this study.

\section{References}

1. Buckner JC: Factors influencing survival in high-grade gliomas. In: Seminars in oncology Elsevier, pp10-14, 2003.

2. Dubrow R, Darefsky AS, Jacobs DI, et al: Time trends in glioblastoma multiforme survival: the role of temozolomide. Neuro Oncol 15: 1750-1761, 2013.

3. Liu Z, Niu Y, Xie M, Bu Y, Yao Z and Gao C: Gene expression profiling analysis reveals that DLG3 is down-regulated in glioblastoma. J Neurooncol 116: 465-476, 2014.

4. Saggioro FP, Neder L, Stávale JN, et al: Fas, FasL and cleaved caspases 8 and 3 in glioblastomas: a tissue microarray-based study. Pathol Res Pract 210: 267-273, 2014.

5. Aghi M and Chiocca EA: Gene therapy for glioblastoma. Neurosurg Focus 20: E18-E18, 2006.

6. Mischel PS, Cloughesy TF and Nelson SF: DNA-microarray analysis of brain cancer: molecular classification for therapy. Nat Rev Neurosci 5: 782-792, 2004.

7. Chen JX, Lu YC, Luo C, et al: Expression and function of differentially expressed genes in glioblastoma by using cDNA microarray. AJSMMU 25: 498-502, 2004.

8. ZhaoZ,Lu Y,Chen J,Hou L-jHu G-h,Luo C: Using Bioinformatics Method to Investigate the Genes Related to Chemosensitivity in Human Glioblastoma. Prog Mod Biomed 19: 003, 2011.

9. Fujita A, Sato JR, Rodrigues LO, Ferreira CE and Sogayar MC: Evaluating different methods of microarray data normalization. BMC Bioinformatics 7: 469, 2006.

10. Gentleman R, Carey V, Huber W, Irizarry RA and Dudoit S: Bioinformatics and computational biology solutions using R and Bioconductor. Springe, 2005.

11. Szekely GJ and Rizzo ML: Hierarchical clustering via joint between-within distances: Extending Ward's minimum variance method. J Classif 22: 151-183, 2005.

12. Deza MM and Deza E: Encyclopedia of distances. Springer, 2009.

13. Obayashi T, Hayashi S, Shibaoka M, Saeki M, Ohta H and Kinoshita K: COXPRESdb: a database of coexpressed gene networks in mammals. Nucleic Acids Res 36: D77-D82, 2008.

14. Janaki C and Joshi RR: Motif detection in Arabidopsis: Correlation with gene expression data. In Silico Biol 4: 149-161, 2004.

15. Smoot ME, Ono K, Ruscheinski J, Wang PL and Ideker T: Cytoscape 2.8: new features for data integration and network visualization. Bioinformatics 27: 431-432, 2011.

16. Huang da W, Sherman BT and Lempicki RA: Systematic and integrative analysis of large gene lists using DAVID bioinformatics resources. Nat Protoc 4: 44-57, 2009.

17. Dudoit S, Shaffer JP and Boldrick JC: Multiple hypothesis testing in microarray experiments. Stat Sci 18: 71-103, 2003.

18. Kanehisa M, Goto S, Hattori M, et al: From genomics to chemical genomics: new developments in KEGG. Nucleic Acids Res 34: D354-D357, 2006.

19. Kanehisa M, Goto S, Kawashima S, Okuno Y and Hattori M: The KEGG resource for deciphering the genome. Nucleic Acids Res 32: D277-D280, 2004.

20. Stower H: Gene regulation: Resolving transcription factor binding. Nat Rev Genet 13: 71-71, 2011.

21. Han L, Suzek TO, Wang Y and Bryant SH: The text-mining based PubChem bioassay neighboring analysis. BMC Bioinformatics 11: 549, 2010.

22. Ohgaki H and Kleihues P: Epidemiology and etiology of gliomas Acta Neuropathol 109: 93-108, 2005.

23. Wechsler-Reya R and Scott MP: The developmental biology of brain tumors. Annu Rev Neurosci 24: 385-428, 2001.

24. Shiina T, Inoko H and Kulski J: An update of the HLA genomic region, locus information and disease associations: 2004. Tissue Antigens 64: 631-649, 2004. 
25. Jäger E, Chen YT, Drijfhout JW, et al: Simultaneous humoral and cellular immune response against cancer-testis antigen NY-ESO-1: Definition of human histocompatibility leukocyte antigen (HLA)-A2-binding peptide epitopes. J Exp Med 187: 265-270, 1998

26. Palucka K, Ueno H and Banchereau J: Recent developments in cancer vaccines. J Immunol 186: 1325-1331, 2011.

27. Neefjes J, Jongsma ML, Paul P and Bakke O: Towards a systems understanding of MHC class I and MHC class II antigen presentation. Nat Rev Immunol 11: 823-836, 2011.

28. Boudjema A, Petit-Teixeira E, Cornelis F and Benhamamouch S HLA-DMA and DMB genes in rheumatoid arthritis. Tissue Antigens 79: 155-156, 2012.
29. Kiepiela P, Leslie AJ, Honeyborne I, et al: Dominant influence of HLA-B in mediating the potential co-evolution of HIV and HLA. Nature 432: 769-775, 2004.

30. Wei J, Barr J, Kong LY, et al: Glioma-associated cancer-initiating cells induce immunosuppression. Clin Cancer Res 16: 461-473, 2010.

31. Machulla HK, Steinborn F, Schaaf A,Heidecke V and Rainov NG: Brain glioma and human leukocyte antigens (HLA)-is there an association. J Neurooncol 52: 253-261, 2001.

32. Moreau A, Varey E, Anegon I and Cuturi MC: Effector mechanisms of rejection. Cold Spring Harb Perspect Med 3: a015461, 2013.

33. Le Moine A, Goldman M and Abramowicz D: Multiple pathways to allograft rejection. Transplantation 73: 1373-1381, 2002. 